\title{
Medienregulierung als objektiv-rechtlicher Grundrechtsauftrag
}

\author{
Wolfgang Hoffmann-Riem
}

Die Kommunikationsfreibeit enthält nicht nur subjektive Rechte des Einzelnen gegen den Staat, sondern auch einen objektiv-rechtlichen Auftrag an den Staat, durch die Rechtsordnung darauf hinzuwirken, dass dieses Grundrecht als Freibeit individueller und kollektiver kommunikativer Entfaltung und politischer Teilhabe durch alle real nutzbar ist. Die strukturellen Veränderungen im Übergang zur Informationsgesellschaft zeigen, dass die bisherige Verankerung subjektiver Rechte nicht ausreicht, um dieses Ziel zu verwirklichen. Deshalb ist eine Rückbesinnung auf den objektiv-rechtlichen Grundrechtsauftrag wichtig, der schon am Beginn der Grundrechtsentwicklung stand. Den Schutz des Rechts benötigen vor allem Machtschwache.

Keywords: Kommunikationsfreiheit, Medienregulierung, Konvergenz, objektiv-rechtlicher Grundrechtsauftrag, regulierte Selbstregulierung, Zensur

Der freiheitliche und demokratische Rechtsstaat der Gegenwart ist ohne Kommunikationsfreiheiten nicht denkbar. Meinungs- und Medienfreiheit (Art. 5 Abs. 1, 2 GG) sind rechtliche Garantien für eine Gesellschaft, die auf der Idee der Handlungsautonomie und Willensbildungsfreiheit ihrer Mitglieder aufbaut. Zugleich wird staatliches Handeln dadurch legitimiert, dass die Ausübung staatlicher Kompetenzen öffentlich zugänglich ist und damit durch Zustimmung und Kritik begleitet und beeinflusst werden kann. Aber auch der nichtstaatliche Bereich gesellschaftlichen Handelns der Bürgerinnen und Bürger ist durch Interaktion und damit Kommunikation geprägt, für die das Recht einen Rahmen bereitstellt, der in einer Demokratie ebenfalls auf Freiheitlichkeit der kommunikativen Selbstverwirklichung ausgerichtet sein muss.

\section{A. Konzeptionelles zur Kommunikationsfreiheit als Grundrecht}

\section{Die Freiheitsidee}

Die Kommunikationsfreiheiten beruhen auf der gleichen Idee wie die anderen liberalrechtsstaatlichen Freiheiten des Grundgesetzes. Es ist die Idee der Freiheit für alle. Die Freiheit wird also kombiniert mit Gleichheit. Freiheit soll das Leben aller prägen, nicht etwa nur - historisch - dem bevorrechtigten Adelsstand oder sonstigen Ständen oder aktuell - einzelnen Bevölkerungsgruppen oder einflussreichen Unternehmen dienen. Erkämpft wurde diese Freiheit im Zuge der so genannten Aufklärung, also in der Neuzeit, beginnend mit dem 18. Jahrhundert ${ }^{1}$. Letztlich verwirklicht wurde sie erst im 20. Jahrhundert.

* Bei diesem Artikel handelt es sich um eine gekürzte und überarbeitete Fassung des Beitrages „Herausforderungen der Kommunikationsfreiheit an das Recht“ aus: Hoffmann-Riem, Kommunikationsfreiheiten, 2002.

1 Zur historischen Entwicklung der Grundrechtsidee s. statt vieler Grimm, Deutsche Verfassungsgeschichte, 1776-1866, 1988; Wabl, Rechtliche Wirkungen und Funktionen der Grund- 
Die Umsetzung der neuen Idee der Freiheit für alle war im 18. und 19. Jahrhundert mit dem Kampf um eine vollständige Veränderung der gesellschaftlichen Verhältnisse verbunden. Zum Aufbau der neuen Ordnung mussten die überkommenen Machtstellungen, insbesondere die der Fürsten und der Kirchen, gebrochen werden, die Schichtung der Bevölkerung in verschiedene Stände musste aufgehoben werden und dies alles musste parallel $\mathrm{zu}$ erheblichen sozialen und technologischen sowie wirtschaftlichen Umbrüchen geschehen. Die revolutionären Bemühungen zielten auf eine neue Struktur der Gesellschaft sowie eine Neuordnung des Verhältnisses zwischen dem Bereich des Staates einerseits und dem der gesellschaftlichen Freiheit andererseits. ${ }^{2}$ Die Freiheitsrechte waren ein Mittel dazu.

Der Freiheitsgebrauch des Einen führt leicht zur Freiheitsminderung des Anderen. Wenn also Freiheit für alle gelten soll, muss es sich um eine geordnete Freiheit handeln. Ein wichtiges Mittel dieser Ordnung ist das Gesetz. Neben der Aufgabe, die Macht der Regierung und Verwaltung zu begrenzen, soll Recht dazu beitragen, die Freiheit vieler miteinander vereinbar zu machen. Herzustellen ist eine Balance zwischen der Macht des Staates als Ordnungskraft einerseits und der Freiheit der Gesellschaft andererseits, aber auch eine Balance zwischen der Freiheitsausübung der verschiedenen Mitglieder der Gesellschaft.

In diesem Sinne sind die Freiheitsrechte von Anfang ihrer historischen Entwicklung an ein Auftrag an den Gesetzgeber, eine freiheitliche Ordnung zu schaffen. In moderner Terminologie lässt sich dies so formulieren, dass die Freiheitsrechte nicht nur subjektive Rechte der Träger der Freiheit vermitteln sollten, sondern zugleich einen objektiv-rechtlichen Auftrag zur Gestaltung der Lebensverhältnisse durch Recht enthielten. ${ }^{3}$ Das ist auch heute noch so. Die folgenden Überlegungen sollen verdeutlichen, dass in der aktuellen Phase des Übergangs zur Informationsgesellschaft die Rückbesinnung auf den objektiv-rechtlichen Grundrechtsauftrag besonders wichtig ist.

\section{Subjektive Rechte und der objektiv-rechtliche Auftrag der Grundrechtsnormen}

\section{Zum Verbältnis subjektiv-und objektiv-rechtlicher Verbürgungen}

Die heute bei der Berufung auf Grundrechte übliche Konzentration des Blicks auf subjektive Rechte darf nicht diese zweite Dimension der Freiheitsrechte vergessen lassen, nämlich die Zuordnung von Staat und Gesellschaft und die Ordnung der Verhältnisse innerhalb der Gesellschaft nach dem Prinzip größtmöglicher gesellschaftlicher und individueller Freiheit. An dem historischen Anfang der Umsetzung der Idee der Freiheit stand in Deutschland nicht das subjektive Recht, sondern das Programm zur Ver-

rechte im deutschen Konstitutionalismus des 19. Jahrhunderts, in: Böckenförde, Moderne Verfassungsgeschichte, 1981, 346 ff.; W. Schmidt, Grundrechtstheorie im Wandel der Verfassungsgeschichte, Jura 1983, 169 ff.; Pieroth, Geschichte der Grundrechte, Jura 1984, 568 ff.; Hufen, Entstehung und Entwicklung der Grundrechte, NJW 1999, 1504 ff.

2 Zur historischen Entwicklung und ihre Analyse s. Grimm, Die Zukunft der Verfassung, 2. Aufl. 1994, $71 \mathrm{ff}$.

3 Grundsätzlich dazu Dreier, Dimensionen der Grundrechte. Von der Wertordnungsjudikatur zu den objektiv-rechtlichen Grundrechtsgehalten, 1993. Zur Darstellung des Verhältnisses subjektiv- und objektiv-rechtlicher Grundrechtsdimensionen s. statt vieler Jarass, Die Grundrechte: Abwehrrechte und objektive Grundsatznormen, in: Badura/Dreier (Hrsg.), Festschrift 50 Jahre Bundesverfassungsgericht, Bd. 1, 2001, 35 ff. 
wirklichung von Freiheit, also die objektiv-rechtliche Dimension. ${ }^{4}$ Dass wir dies heute weitgehend außer Acht lassen und uns auf das subjektive Recht konzentrieren können, ist ein Beleg dafür, dass das objektiv-rechtliche Programm unter den bisherigen Rahmenbedingungen weitgehend erfüllt ist, der Gesetzgeber also den Auftrag zur Schaffung einer freiheitlichen Ordnung verwirklicht hat. Im Kontext dieser Ordnung gibt es subjektive Rechte und in der Folge scheint die objektiv-rechtliche Grundlage nicht mehr so wichtig zu sein. Ist die Freiheitsordnung eingerichtet und haben die einzelnen Bürger subjektive Rechte, dann genügt es für die juristische Freiheitsverwirklichung, solche subjektiven Rechte zu verfolgen. Wenn sich die Verhältnisse aber - wie gegenwärtig grundlegend ändern, erhält die Einsicht in die ebenfalls mit dem Grundrecht verbundene andere Dimension eine neue Bedeutung. Diese objektiv-rechtliche Dimension und ihr Zusammenspiel mit der subjektiv-rechtlichen Komponente des Freiheitsschutzes hat das Bundesverfassungsgericht schon früh herausgearbeitet und vorrangig anhand der Meinungs- und Medienfreiheit entwickelt.

Das ist schon an der Basisentscheidung zum Verhältnis der Kommunikationsfreiheit und der privatrechtlich geschützten Entfaltungsfreiheiten anderer ablesbar, dem LüthUrteil. ${ }^{5}$ Es ging um einen Boykottaufruf, der nach Auffassung des Bundesverfassungsgerichts durch die Kommunikationsfreiheit geschützt war, obwohl Grundrechte anderer - die des Regisseurs und der Verleihfirma des boykottierten Films - beeinträchtigt wurden. Die Kommunikationsfreiheit wurde vom BVerfG nicht nur als subjektives Recht zur Abwehr von staatlichen Eingriffen in die Freiheit verstanden. Sie strahlte vielmehr wegen ihrer grundsätzlichen Bedeutung als objektiv-rechtliche Position auch in andere Regelungen, insbesondere in den Bereich zivilrechtlicher Beziehungen, hinein, und musste deshalb bei der Ausfüllung der unbestimmten Rechts-(Wert-)Begriffe des BGB berücksichtigt werden. Das Gesetz (hier das BGB) wurde als Mittel der Umsetzung objektiv-rechtlicher Vorgaben in subjektive Rechte verstanden, hier allerdings nicht im Zuge neuer Rechtsetzung, sondern der Auslegung und Anwendung vorhandenen Rechts. Inhaltlich wurde als entscheidend für die Lösung des konkreten Konflikts angesehen, dass Lüth eine Angelegenheit von öffentlichem Interesse verfolgte, also nicht aus Eigennutz handelte, insbesondere nicht von einem Gewinninteresse getrieben war. Lüth forderte nämlich nicht zum Boykott eines Films auf, weil er ein Konkurrent des benachteiligten Regisseurs Veith Harlan oder dessen Vertriebsfirma war und etwa den Vertrieb eines eigenen Filmes fördern wollte. Es ging ihm lediglich darum, das öffentliche Gewissen anzusprechen und das öffentliche Bewusstsein auf eine Gefahr für die Freiheit (das Wiederaufkommen nationalsozialistischen Gedankenguts) zu richten. Diese Motivlage hat das Gericht akzeptiert und für die Abwägung bei der Zuordnung kollidierender Rechte entschieden, der Rechtsstreit zwischen den Bürgern müsse unter Rückgriff auf die objektiv-rechtliche Ordnung der Grundrechte bewältigt werden.

Dies bedeutet nicht, dass die Meinungsfreiheit nun alle anderen Aspekte überwiegt. Wohl aber muss das Grundrecht der Meinungsfreiheit bei einem Konflikt mit anderen Grundrechten - etwa der Film- und Kunstfreiheit oder der ökonomischen Entfaltungsfreiheit - hinreichend berücksichtigt werden. In dem erforderlichen Abwägungsprozess ist nach einem Weg des angemessenen Ausgleichs zu suchen, der den verschiedenen Freiheitsrechten möglichst große Wirksamkeit verleiht, dabei aber auch ihre Bedeutung für 
die Verwirklichung von Verfassungsprinzipien wie dem der Demokratie gerecht wird.

Auf die gleiche argumentative Basis, die objektiv-rechtliche Grundlegung der Grundrechte, hat das BVerfG auch seine Rechtsprechung zur Medienfreiheit, insbesondere zur Sicherung der Funktionsfähigkeit der Rundfunkordnung, gestützt. Mehrere Rundfunkurteile geben davon Rechenschaft, beispielhaft das FRAG-Urteil und das Niedersachsen-Urteil. ${ }^{6}$ Gegenstand verfassungsrechtlicher Prüfung waren hier nicht Kollisionen unterschiedlicher Rechtsgüter, sondern gesetzliche Regelungen, die darauf zielten, die Ordnung der Massenkommunikation, insbesondere des Rundfunks, an dem für einen demokratischen Rechtsstaat wichtigen Ziel der Vielfaltsicherung auszurichten und eine auf dieses Ziel bezogene Funktionsfähigkeit der Medienordnung gesetzlich abzusichern.

In rechtsdogmatischer Hinsicht verweist dies auf die so genannten Ausgestaltungsgesetze, die das Gericht von den eine Kollision unterschiedlicher Rechtspositionen bewältigenden Schrankengesetzen unterscheidet. ${ }^{7}$ Die Besonderheit von Ausgestaltungsgesetzen wird darin gesehen, dass mit ihrer Hilfe die Art der Kommunikationsversorgung der Gesellschaft insgesamt und die Möglichkeit für alle am Kommunikationsprozess Interessierten, sich kommunikativ $\mathrm{zu}$ entfalten, gesichert werden soll. Zur Verwirklichung dieses Ziels können subjektive Rechte der Kommunikatoren und Rezipienten beitragen; ihre Einrichtung allein reicht aber nicht zur Freiheitssicherung. Vielmehr muss durch eine „positive Ordnung“ die Vielfalt der Kommunikation sichergestellt werden. ${ }^{8}$ Die Medien sollen in die Lage versetzt werden, einerseits bei der Vermittlung der in der Gesellschaft gebildeten Fakten und Werte an die Öffentlichkeit mitzuwirken (also „Medium“ zu sein), daneben aber auch eine aktive, durchaus eigenständig wertende und beeinflussende Funktion im Meinungsbildungsprozess wahrzunehmen (also „Faktor“ der Meinungsbildung zu sein).

In solchen Ausführungen und den zugrunde gelegten grundrechtsdogmatischen Konstruktionen wird maßgebend, dass Kommunikationsfreiheit eine über den Schutz individueller Entfaltung hinausgehende Bedeutung hat. Kommunikation ist ein Lebensnerv einer Demokratie und eines Rechtsstaats, die Kommunikationsfreiheit Voraussetzung ihrer Funktionsfähigkeit.

Das liberale Konzept der Kommunikationsfreiheit versprach sich aus dem freien Austausch von Tatsachen und Meinungen die Fähigkeit zur Meinungsbildung und zur kommunikativen Orientierung und dabei auch zur Erkenntnis des „Richtigen“ und sah diese Funktion als notwendige Grundbedingung einer Gesellschaft, die ihre Angelegenheiten in Eigenverantwortung regelt. Heute sind Zweifel angebracht, ob und wieweit diese Konzeption noch trägt, und es muss nach einem neuen Konzept gesucht werden, das sich weniger an der Vorstellung der Wahrheitsfindung durch Diskurs oder gar an dem Bild des „Marktplatzes der Meinungen“ orientiert, sondern stattdessen die große Bandbreite unterschiedlicher Verwendungszusammenhänge von Kommunikation, die Vernetzungen unterschiedlicher Kommunikationswelten und die besonderen Bedingungen

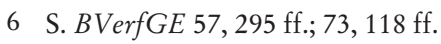

7 Zum Unterschied von Ausgestaltungs- und Schrankengesetzen s. BVerfGE 73, 118, 166; 90, 145, 172; Ruck, Zur Unterscheidung von Ausgestaltungs- und Schrankengesetzen im Bereich der Rundfunkfreiheit, AöR 117 (1992), 543 ff.; Rossen, Freie Meinungsbildung durch den Rundfunk, 1988, $285 \mathrm{ff}$., $307 \mathrm{ff}$.

8 BVerfGE 57, 295, 320. 
einer durch Fragmentierung und Pluralisierung geprägten Gesellschaft verarbeitet. ${ }^{9}$ Ungeachtet des Auftrags, das Konzept der Kommunikationsfreiheit auf die jeweiligen Rahmenbedingungen der Gesellschaft abzustimmen, kann jedoch festgestellt werden, dass kommunikative Entfaltung weiterhin als konstitutiv für den gesellschaftlichen und politischen Prozess angesehen wird. Allerdings galt es im bürgerlich-liberalen Verfassungsstaat als hinreichend, im Rahmen des rechtlich Regelbaren die Möglichkeiten subjektiver kommunikativer Entfaltung abzusichern, also auf die Selbstregulierungskraft kommunikativer Prozesse zu vertrauen. Im 20. und beginnenden 21. Jahrhundert ist dieses Vertrauen keineswegs ungebrochen. Einsichten in die Manipulierbarkeit kommunikativer Prozesse, in die Herausbildung von immensen Machtpositionen in einer marktgesteuerten Gesellschaft und in die Potenziale des Machtmissbrauchs auch durch gesellschaftliche Machtträger haben dazu geführt, dem Recht eine weitaus größere Rolle zuzuschreiben. Zugleich aber gibt es Anhaltspunkte, dass das Recht in der globalen und vielfältig vernetzten Kommunikationswelt der Gegenwart allenfalls begrenzt geeignet ist, entsprechende Gefährdungen einzudämmen oder auszuschließen (s. auch u. B I-VI).

Die im liberalen Modell erhoffte Möglichkeit zur individuellen und sozialen Entfaltung lässt sich durch Grundrechte, wenn sie als bloße Abwehrrechte gegen den Staat zu verstehen sind, schon allein deshalb nicht hinreichend sichern, weil die Kommunikationsfreiheiten in der Ordnung des Grundgesetzes in ein System von verschiedenen verfassungsrechtlichen Zielwerten und Verbürgungen eingeordnet sind, die der Staat in ihrer Funktionsweise ebenfalls gewährleisten muss. Unter Berücksichtigung der Staatszielbestimmungen Rechtsstaat, Demokratie und Sozialstaat, die auch auf die Ordnung der Gesellschaft zurückwirken, wird Kommunikation (auch) mit dem Blick auf die Entwicklung von Staat und Gesellschaft geschützt. Soweit die Funktionsfähigkeit einer Demokratie auf die Leistungsfähigkeit von Kommunikationsprozessen und die eines Rechtsstaats auf die rechtliche - und zwar rechtlich angemessene - Ordnung von Lebensverhältnissen angewiesen ist, fordert auch der programmatische Gehalt des Grundgesetzes entsprechende rechtliche Vorgaben zur Sicherung der Idee der Freiheit als einer real wirksamen Freiheit unter den Bedingungen der Gegenwart.

Dies bedeutet allerdings nicht, dass Kommunikation nur in solchen Kontexten, etwa im Rahmen demokratischer Prozesse oder gar politischer Entscheidungen, geschützt wäre. Kommunikationsfreiheiten sichern kommunikative Entfaltung schlechthin, also mit dem Blick auf die Bedeutung von Kommunikation in allen Lebensbereichen, die auf Interaktion angewiesen sind, so in der Berufswelt, der Freizeit oder der Privatsphäre. ${ }^{10}$ Der Bezug auf den Prozess demokratischer Willensbildung und die damit verbundene Notwendigkeit zur Absicherung der Meinungsbildungsfreiheit aller kann allerdings besondere rechtliche Ausgestaltungen erfordern. Die Mediengesetzgebung ist ein Beispiel

9 Zu dieser Problematik s. Hoffmann-Riem, Sozialwissenschaften in der Rechtsanwendung - am Beispiel der Nutzung der Medienforschung in der Rechtsprechung zum Medienrecht, Zeitschrift für Rechtssoziologie, 2001, 3 ff. sowie mit grundsätzlicher Kritik (statt vieler) Vesting, Zur Zukunft und Konstruktion des Medien- und Telekommunikationsrechts in den hybriden Beziehungsnetzwerken der „Informationsgesellschaft“, in: Rossen-Stadtfeld/Wieland (Hrsg.), Steuerung medienvermittelter Kommunikation, 2001, 83 ff.; ders., Soziale Geltungsansprüche in fragmentierten Öffentlichkeiten, AöR 122 (1997), 337 ff.; Ladeur, Meinungsfreiheit, Ehrenschutz und die Veränderung der Öffentlichkeit in der Massendemokratie, AfP 1993, $531 \mathrm{ff}$. S. auch $B \operatorname{Ver} G E 103,44,67$.

10 Eine entsprechende Reichweite betont das BVerfG beispielsweise in $B V \operatorname{erf} G E$ 101, 361, $389 \mathrm{f}$. 
dafür, wenn sie Vielfalt, Manipulationsfreiheit u. ä. abzusichern sucht. Insofern dient sie der Erfüllung des objektiv-rechtlichen Gestaltungsauftrags der Grundrechte in Verbindung mit den Verfassungsprinzipien, zielt aber gleichwohl auf die Absicherung subjektiver kommunikativer Entfaltung. In diesen Kontext gehört die Formulierung des Bundesverfassungsgerichts, dass der objektiv-rechtliche Grundrechtsgehalt dem subjektiven Grundrechtsschutz diene. ${ }^{11}$

\section{Risiken staatlicher Aufgabenwabrnebmung}

Eine solche Rechtsprechung ist von einem großen Vertrauen in die Funktionsfähigkeit von Rechtsstaat und Demokratie geprägt, denn sie gilt nicht vorrangig der Abwehr staatlichen Machtmissbrauchs - dazu sind die Grundrechte allerdings weiterhin einsetzbar -, sondern versteht den Staat auch als Garanten des Schutzes vor privat-gesellschaftlichem Machteinsatz, insbesondere Machtmissbrauch. Dieses verfassungsrechtlich vorausgesetzte Vertrauen in den Staat und seine Organe setzt nicht nur hinreichende verfahrensmäßige Sicherungen der Bändigung staatlicher Machtausübung (rechtsstaatliche Verfahren, öffentliche Kritik, Unabhängigkeit der zur Kontrolle befugten Gerichte u. ä.) voraus, sondern auch materiellrechtliche Gebote, so das staatlicher Inhaltsneutralität. ${ }^{12}$

Das Neutralitätsgebot gilt bei der Ausgestaltung der Medienordnung ebenso wie bei der Bewältigung konkreter Kollisionslagen im Rahmen von Schrankengesetzen. So darf der Staat nicht bestimmte Inhalte als erwünscht und andere als unerwünscht definieren. Allerdings bedeutet der weit gezogene Schutzbereich des Art. 5 GG nicht, dass Art und Weise des Schutzes vollständig von dem Kontext abstrahieren, in dem eine Kommunikation erfolgt, soweit sie zugleich Rechte anderer beeinträchtigt. Ob ein kommunikatives Verhalten in den Schutzbereich des Grundrechts fällt, ist zwar von ihrem Inhalt unabhängig. Ob und wieweit sie aber in Kollisionslagen zum Schutz anderer Rechtsgüter zurückzutreten hat, hängt demgegenüber von weiteren Feststellungen ab. Rechtsdogmatischer Anknüpfungspunkt ist insbesondere die bei der Prüfung der Verhältnismäßigkeit einer Beschränkung erfolgende Klärung der Angemessenheit einer Zuordnung von Mittel und Zweck (Verhältnismäßigkeit i.e.S.). Bei der Prüfung der Angemessenheit wird aus der - in der Literatur allerdings umstrittenen ${ }^{13}$ - Sicht des Bundesverfassungsgerichts z. B. bedeutsam, ob die beanstandete Kommunikation „in der Sorge um politische, wirtschaftliche und soziale oder kulturelle Angelegenheiten der Allgemeinheit" erfolgt bzw. „der Einwirkung auf die öffentliche Meinung“ dient. ${ }^{14}$ Dann ist sie stärker geschützt als wenn mit ihr nur gewerbliche oder sonst wie persönliche Interessen verfolgt werden. Auch darf, etwa bei der Abwägung mit kollidierenden Persönlichkeitsrechten, berücksichtigt werden, „ob Fragen, die die Öffentlichkeit wesentlich an-

$11 B \operatorname{VerfGE}$ 57, 295, 320. Dort hebt das Gericht das Wort „dienende“ Freiheit ausdrücklich hervor.

12 Es ist verknüpft mit dem Gebot der Staatsfreiheit der Medien. Dazu vgl. Ossenbühbl, Rundfunk zwischen Staat und Gesellschaft, 1975; Jarass, Die Freiheit der Massenmedien, 1978, 198 ff.; Gersdorf, Staatsfreiheit des Rundfunks in der dualen Rundfunkordnung der Bundesrepublik Deutschland, 1991, $89 \mathrm{ff}$.

13 S. dazu statt vieler Lerche, in Festschrift für G. Müller 1970, 197 ff.; Schmitt Glaeser NJW 1996, 873, 874 ff.; Neben, Triviale Personenberichterstattung als Rechtsproblem, 2001.

$14 B \operatorname{VerfGE} 62,230,244 ; 85,1,16$. 
gehen, ernsthaft und sachbezogen erörtert oder lediglich private Angelegenheiten, die nur die Neugier befriedigen, ausgebreitet werden “. ${ }^{15}$ Hier versucht das Gericht eine Antwort auf neuartige Erscheinungen und damit verbundene Gefährdungen, wie die Kommerzialisierung vieler Lebensbereiche und die damit einhergehende Tendenz der Medien zur massiven Personalisierung und Skandalisierung ${ }^{16}$, die insbesondere im Rahmen einer vorrangigen Orientierung an Unterhaltungsinteressen der Konsumenten beobachtbar ist. Am Beispiel des Persönlichkeitsrechts lassen sich problematische Folgen dieser Kommerzialisierung gut beobachten, sei es bei Verletzungen von Persönlichkeitsrechten mit dem Ziel privatwirtschaftlicher Gewinnerzielung - etwa in der Boulevardpresse -, aber auch bei der Vermarktung der eigenen Persönlichkeitsrechte Prominenter. ${ }^{17}$ Das Recht ist in einer vorrangig marktwirtschaftlichen, also vorrangig kommerziell geprägten Gesellschaftsordnung zwar nicht als Mittel einsetzbar, die Kommerzialisierung zu verhindern, wohl aber kann es dazu dienen, in Kollisionsfällen gegenläufigen Interessen Verwirklichungschancen zu belassen. Dies setzt regelmäßig eine Güterabwägung und Zuordnung der kollidierenden Güter in einer Weise voraus, die allen betroffenen Interessen optimale Verwirklichungsmöglichkeiten belässt. Der entsprechende Vorgang wird - in Anlehnung an Konrad Hesse ${ }^{18}$ - als Herstellung „praktischer Konkordanz“ bezeichnet.

Abwägungen zwischen verschiedenen kollidierenden Rechtsgütern können allerdings eine Einbruchstelle subjektiver Bewertungen oder gar missbräuchlicher Zuordnungen sein. Weil die Machtausübung durch den Staat, anders als meist der Machtgebrauch durch private Unternehmen, seinerseits einer Vielzahl von Kontrollen unterliegt und weil die letztlich entscheidenden staatlichen Gerichte aufgrund ihres besonderen Status' der Unabhängigkeit vergleichsweise gute (wenn auch nicht absolut sichere) Voraussetzungen für die Vermeidung von Machtmissbrauch bieten, gilt das mit jeder Abwägung verbundene Risiko als hinnehmbar. Die Alternative, der Verzicht auf die wertende Zuordnung der miteinander kollidierenden Rechtsgüter, liefe Gefahr, denjenigen Schutz zu verweigern, die ihn ohne Hilfe des (ausgleichenden) Rechts nicht erreichen könnten. Die Sicherung realer Grundrechtsverwirklichung zugunsten aller von Beeinträchtigungen durch andere Rechtsträger Betroffenen lässt sich unter den komplexen, vielfach vernetzten Lebensverhältnissen der Gegenwart nicht ohne Vertrauen in eine Instanz erreichen, die gegebenenfalls auch als Gegenmacht gegen gesellschaftliche Machtträger zu wirken vermag.

War dies früher der mit Hoheitsgewalt ausgestattete Nationalstaat, so werden es zukünftig vermehrt auch die mit Hoheitsgewalt ausgestatteten Träger des supranationalen Verbunds der Europäischen Gemeinschaft sein. Derartige Hoheitsträger sind als Garanten der Freiheit weiterhin unverzichtbar. Dies bedeutet nicht, dass Hoheitsträger nicht weiterhin auch als Gefährder von Freiheit auftreten können. Die Abwehr hoheit-

15 BVerfGE 101, 361, 391. Diese schon mehrfach vom BVerfG benutzte Formulierung ist nicht voll geglückt, da sie Fragen des Informationsgegenstandes (öffentliche/private Angelegenheiten) mit denen der Art der Darstellungsweise bzw. dem Darstellungsinteresse (ernsthaft, sachbezogen oder nicht) vermengt und damit nicht zu einer tragfähigen Gegensatzbildung kommt.

$16 \mathrm{Zu}$ solchen Erscheinungen vgl. Weiß, Fern-Sehen im Alltag, 2001; Groebel u. a., Bericht zur Lage des Fernsehens für den Präsidenten der Bundesrepublik Deutschland, 1995.

17 S. dazu BVerfGE 101, 361, 385.

18 S. Hesse, Grundfragen des Verfassungsrechts der Bundesrepublik Deutschland, 20. Aufl. 1995/ 2000, Rn. $317 \mathrm{ff}$. 
lichen Machtmissbrauchs bleibt eine wichtige Aufgabe des Rechts. Die Abwehrfunktion ist in der Rechtsordnung und -anwendung sowie der Wissenschaft allerdings ausgiebig ausgearbeitet worden. Hier gibt es weniger Unsicherheiten als bei der Verwirklichung der im Folgenden weiter zu betrachtenden Gewährleistungsfunktion.

\section{B. Aktuelle Herausforderungen}

Kommunikationsfreiheit als Freiheit individueller und kollektiver kommunikativer Entfaltung und als Freiheit politischer Teilhabe ist auf Rahmenbedingungen angewiesen, die möglichst allen die Chance der realen Ausübung dieser Freiheitsrechte einräumen. Dies erfordert auch die Vorsorge dafür, dass die gesellschaftlichen Machtverhältnisse sich nicht als Verhinderung folgenreicher Kommunikationsteilhabe der Machtschwachen auswirken. Soweit die Funktionsfähigkeit der Kommunikationsordnung auf rechtliche Vorkehrungen angewiesen ist, kann der objektiv-rechtliche programmatische Auftrag an den Staat - jetzt auch an die Organe der EG ${ }^{19}$ - zur Sicherung dieser Funktionsbedingungen eine darauf gerichtete Normenordnung fordern. Auch insoweit behält der objektiv-rechtliche Auftrag die erwähnte dienende Funktion gegenüber subjektiven Rechten der Kommunikationsteilhabe.

Das auf Vielfaltsicherung gerichtete Medien-, insbesondere Rundfunkrecht ist ein prominentes Beispiel des Versuchs der Funktionssicherung (s. u. II). Weil das staatliche Recht Machtbegrenzungsrecht ist, kann es nicht überraschen, dass die Setzung von besonderem Medienrecht umstritten ist und besonders umstritten in den achtziger Jahren war, als sich die aktuellen Umbrüche der Medienlandschaft zu verwirklichen begannen. ${ }^{20}$ Auf die Fragen des Regelungsbedarfs und der Regelungsmöglichkeit sei daher ein besonderes Augenmerk gerichtet.

\section{Entwicklungen in der Informationsgesellschaft}

Die aktuellen Herausforderungen der Meinungs- und Medienfreiheit beruhen insbesondere auf den Neuerungen der Informationsgesellschaft. Derartige Neuerungen zeigen sich insbesondere in den Informationstechnologien und der Computertechnik - insbesondere der Digitalisierung und der Möglichkeit der Datenkompression und -dekompression -, dem Aufbau neuer Netzinfrastrukturen, der Entwicklung neuer Endgeräte und, verbunden mit diesen Änderungen, der Entstehung neuer technischer und inhaltlicher Kommunikationsdienste. Dies revolutioniert die gesamte Kommunikationsordnung, und zwar nicht nur in der Individual-, sondern auch in der Massenkommunikation.

Erweiterte publizistische und ökonomische Entfaltungschancen sind mit neuen Pfaden der Verbreitung von Individual- und Massenkommunikation (etwa über das Internet) und neuen Inhalten (Programmen/Diensten) und neuen Vermarktungsformen verbunden. Dies wirkt auf die Rezeptionsmöglichkeiten und -gewohnheiten zurück.

19 Auch die europarechtlich verankerten Grundrechte enthalten objektiv-rechtliche Elemente, s. dazu Kübling, Die Kommunikationsfreiheit als europäisches Gemeinschaftsgrundrecht, 1999 sowie ders., Grundrechtskontrolle durch EuGH, EuGRZ 1997, 296 ff.

20 Zur Entwicklung s. Eifert/Hoffmann-Riem, Die Entstehung und Ausgestaltung des dualen Rundfunksystems, in: Schwarzkopf (Hrsg.), Rundfunkpolitik in Deutschland, Bd. 1, 1999, $50 \mathrm{ff}$. 
Unter dem Schlagwort „Konvergenz“21 wird das zunehmende wechselseitige Durchdringen der Informationstechnologie -, Telekommunikations- und Medienmärkte sowie der Geräte, Netzinfrastrukturen und technischen sowie inhaltlichen Dienste thematisiert. Neue Anbieter treten auf und alte wie neue Akteure versuchen, in den verschiedenen Segmenten der Multimedia-Märkte erfolgreich zu sein. Die Rede ist von Multimedia-Wertschöpfungsketten oder - um die Dynamik besser zum Ausdruck zu bringen - von Multimedia-Wertschöpfungsnetzwerken. ${ }^{22}$

Mit solchen Prozessen ist die Umstrukturierung bisheriger Tätigkeiten verbunden, deutlich sichtbar nicht nur bei neuen Medien- und Telediensten, sondern selbst bei dem klassischen Rundfunk. War die Veranstaltung von Rundfunk - verstanden als die Produktion von Programmen, deren redaktionelle Zusammenstellung und die Verbreitung an die Rezipienten - früher ein relativ einheitlicher Vorgang, hat seit langem ein Prozess starker Ausgliederung einzelner Elemente begonnen. Tätigkeiten, die früher zur Rundfunkveranstaltung gezählt wurden, werden in vor-, neben- und nachgelagerte Bereiche ausgelagert. ${ }^{23}$ Zugleich wird versucht, durch Beteiligungen, Allianzen oder sonstige Verträge Verbindungen zwischen den einzelnen Elementen herzustellen. Parallel dazu werden neue Dienste entwickelt, die neue Inhalte entstehen lassen bzw. neue Wege der Verbreitung und Vermarktung eröffnen. In der Folge ist die alte Rundfunkordnung nunmehr nur noch ein Teil einer weit ausgefächerten Kommunikations- und Informationsordnung, die eine Vielzahl der direkt an die Rezipienten gerichteten neuen Dienste, aber auch einen bunten Strauß unterschiedlicher Dienste für andere Unternehmen umfasst. Der Entwicklungsstand dieser „dienstespezifisch diversifizierten Informationsordnung “ 24 wirkt auf die Möglichkeiten der Veranstaltung von Rundfunk und in der Folge der rechtlichen Ausgestaltung der Rundfunkordnung zurück und wirft die Frage auf, ob es erforderlich oder möglich ist, auch die anderen Marktsegmente in eine solche Gesetzgebung einzubeziehen.

Früher war Rundfunkregulierung fast ausschließlich Veranstalterregulierung und konnte dies auch sein, weil auf diese Weise alle vom Veranstalter selbst durchgeführten, jetzt aber der Veranstaltung i.e.S. vor- und nachgelagerten Tätigkeiten umfasst wurden. Würden nunmehr Grundsteine für Funktionsdefizite der Medienordnung in den von der Rundfunkveranstaltung abgelösten vor-, neben- und nachgelagerten Bereichen gelegt, so könnte dies Konsequenzen für die Funktionstauglichkeit der Medienordnung haben. Weitere Probleme werden an der Beobachtung sichtbar, dass die neuen Dienste zum Teil traditionellen Rundfunk substituieren oder doch substituieren können, so dass möglicherweise Gefährdungen der Kommunikationsversorgung und Risiken der machtmissbräuchlichen Einwirkung auf die öffentliche Meinungsbildung auch von solchen Diensten ausgehen können.

Sollen die traditionell mit Art. 5 GG verbundenen Ziele und der Auftrag zur Sicherung einer funktionsfähigen Kommunikationsordnung weiterhin gültig sein - das

21 Dazu s. das „Grünbuch zur Konvergenz der Branchen Telekommunikation, Medien- und Informationstechnologie und ihren ordnungspolitischen Auswirkungen" der EG-Kommission, KOM (97) 623; Hoffmann-Riem/Schulz/Held, Konvergenz und Regulierung, 2000.

22 Dazu s. Zerdick u. a., Die Internet-Ökonomie, 1999.

23 Dazu vgl. H. D. Schröder (Hrsg.), Entwicklung und Perspektiven der Programmindustrie, 1999 m.w.Hinw.

24 So der Titel der von Kops/Schulz/Held herausgegebenen Publikation: Von der dualen Rundfunkordnung zu einer dienstespezifisch diversifizierten Informationsordnung, 2001. 
Grundgesetz stellt dies nicht in Frage -, dann gehört die bisherige Ausgestaltung der Kommunikationsordnung im Interesse des Freiheitsschutzes auf den Prüfstand. Dies aber wirft nicht nur neue Fragen der rechtstechnischen Umsetzung der verfassungsrechtlichen Anforderungen auf, sondern führt zu einigen Grundsatzfragen, wie insbesondere der, ob rechtliche Regulierung angesichts der Vervielfältigung der Dienste und infrastrukturellen Rahmenbedingungen überhaupt noch nötig ist und - falls dies grundsätzlich bejaht wird - in hinreichender Weise erfolgreich sein kann. Damit stellt sich im Kommunikationsbereich die Frage nach der Steuerungskompetenz von Hoheitsträgern neu. Angesichts der Internationalisierung und Globalisierung sowie der Ökonomisierung der Lebensverhältnisse und der vielfältigen Vernetzungen unterschiedlicher Tätigkeiten gibt es vielfältige neue Möglichkeiten des Ausweichens vor hoheitlicher Regulierung. Es wird aber auch grundsätzlich bestritten, dass Regulierung sinnvoll ist, und dann angenommen, der Markt sei im Vergleich zum Staat der bessere Regulator.

\section{Rechtfertigung hoheitlicher Medienregulierung}

Die vom Bundesverfassungsgericht betonten und ebenfalls - wenn auch in abgeschwächter Weise - von der EG anerkannten Ziele einer an Gemeinwohlzwecken orientierten Mediengesetzgebung, insbesondere an der Sicherung von Vielfalt, bestehen fort. ${ }^{25}$ Vielfalt ist in einer pluralen und fragmentierten, auf das Demokratieprinzip verpflichteten Gesellschaft ein wichtiger verfassungsrechtlicher Zielwert. Hoheitliche Regulierung ist allerdings nur gerechtfertigt, wenn ein Risiko der Zielverfehlung besteht und die Regulierung dazu beitragen kann, die Zielerreichung zu fördern.

Die rundfunkrechtliche Regulierung ist historisch als Technikregulierung entstanden. ${ }^{26}$ Es ging darum, das Risiko eines „Chaos im Äther“ durch unbegrenzte und nicht abgestimmte Nutzung von Übertragungsfrequenzen zu vermeiden und Interferenzen auszuschließen. Die Möglichkeit zur staatlichen Zuteilung knapper Ressourcen wurde aber zugleich zur Entwicklung eines politisch und später publizistisch geprägten Zuteilungskriteriums genutzt. Die knappe Übertragungsressource sollte bevorzugt denen zugute kommen, die Inhalte (Programme) über die Netzinfrastrukturen verbreiteten, die nicht nur auf optimale Gewinnerzielung oder die Verfolgung eigener politischer Ziele gerichtet waren, sondern auch der Allgemeinheit nutzten, sich insbesondere publizistischen Belangen widmeten.

Heute ist die Knappheit der Übertragungsmöglichkeiten beseitigt oder - so gegenwärtig bei terrestrischer Übertragung - zumindest vermindert, so dass der Knappheitsbefund keineswegs ausreicht, um Regulierung zu rechtfertigen. Knappheit war allerdings niemals die Rechtfertigung, sondern vielmehr nur der Anknüpfungspunkt staatlicher Regulierung gewesen. Risiken der Verfehlung der Vielfaltsvorgaben sind jedoch auch jenseits von Knappheit zumindest plausibel, so dass es eine nicht an die Knappheit anknüpfende Rechtfertigung von Regulierung geben kann. Die in der Wirtschaftswis-

25 Zur Vielfaltsicherung s. BVerfGE 57, 295, 319 ff. Im Europarecht wird das Vielfaltsziel auch als Bestandteil des öffentlichen Interesses anerkannt, das Beschränkungen der Dienstleistungsfreiheit rechtfertigen kann, s. EuGH, Slg. 1991, I - 4007 sowie I - 4009.

26 Vgl. Binz, Geschichte der deutschen Frequenzverwaltung, ArchPF 1989, 232 ff.; Scherer, Frequenzverwaltung zwischen Bund und Ländern, 1987; Eifert, Grundversorgung mit Telekommunikationsleistungen im Gewährleistungsstaat, 1998. 
senschaft entwickelten Theorien meritorischer Güter ${ }^{27}$, die Auseinandersetzung mit externen Effekten ${ }^{28}$ sowie Einsichten der Informationsökonomie ${ }^{29}$ bieten Erklärungen für Marktdefizite und signalisieren dementsprechend einen Bedarf zur Vermeidung oder Kompensation solcher Defizite durch Regulierung. ${ }^{30}$ Gegenwärtig konzentriert der Regulierungsbedarf sich auf die vielfältigen Zugangsengpässe und -filterungen, die trotz Überwindung der Knappheitslagen bei den Verbreitungstechnologien die Kommunikationsordnung prägen und das Konzept der gleichen Freiheit für alle gefährden. Beobachtbar sind technikbezogene, marktmachtbezogene, angebotsbezogene und rezipientenbezogene Zugangsprobleme. Freiheitsschutz wird notwendig zum Zugangsschutz. ${ }^{31}$

Gegenwärtig konzentriert sich die Aufmerksamkeit exemplarisch auf Zugangshürden im Bereich des digitalen Fernsehens. Dort sind solche Hürden mit einer Reihe so genannter Hilfsdienste verknüpft. Sie beziehen sich insbesondere auf den so genannten conditional access (die Setzung von Konditionen für den Zugang zur Verbreitung von Kommunikationsdiensten, etwa bei Pay-TV), aber auch auf das Multiplexing, die Programmpaketvermarktung (packaging) und insbesondere auf Navigationssysteme, die zur Orientierung und zur Steuerung des Informationsabrufs eingesetzt werden. ${ }^{32} \mathrm{Zu}$ gangsprobleme kann es zum einen für Kommunikatoren (Veranstalter, Anbieter) geben, etwa bei dem Zugang zu Produktionsfaktoren, zu Programmrechten, zu Verbreitungsund Vermarktungsmöglichkeiten; für die Nutzer können die Zugänglichkeit der Netzinfrastruktur und die Verfügbarkeit nicht nur quantitativ, sondern auch qualitativ auf ihre Bedürfnisse abgestimmter Inhaltsangebote problematisch sein; auch bestehen Risiken manipulativen Zugriffs auf ihre Rezeptionsbereitschaft und auf die Nutzung ihrer kommunikativen Kompetenz. Damit sind nur Problemzonen benannt, ohne dass sich schon bestimmte Wege der Problembewältigung anbieten. Diese besteht in der Aufnahme des objektiv-rechtlichen Grundrechtsauftrags zur Gestaltung der Medien- und Informationsordnung der Gegenwart nach dem Prinzip der gleichen Freiheit für alle.

27 Dazu siehe Zerdick (Fn. 22); McKnight/Bailey (eds.), Internet Economics, 1997; Shapiro/Varian, Information Rules: A Strategic Guide to the Network Economy, 1998.

28 Eine aktuelle Auswertung der verschiedenen medienökonomischen Ansätze findet sich bei Kops, Von der dualen Rundfunkordnung zur dienstespezifisch diversifizierten Informationsordnung?, in: Kops/Schulz/Held (Fn. 24); Hoffmann-Riem, Regulierung der dualen Rundfunkordnung, 2000, $120 \mathrm{ff}$.

$29 \mathrm{Zu}$ diesem Maßstab siehe BVerfGE 100,313, 373. Die für Grundrechtseingriffe entwickelte Formel zur Eignung rechtlicher Regelungen zur Zielerreichung passt im Kern auch für Ausgestaltungsgesetze, deren Rechtmäßigkeit daran gemessen wird, dass sie der Sicherung der Funktionsfähigkeit der Medienordnung dienen und „deshalb geeignet sind zu bewirken, was Art. 5 Abs. 1 GG gewährleisten will“ ( $B V \operatorname{erf} G E$ 57, 295, 320).

30 S. ferner die Darstellungen von Heinrich, Medienökonomie, Bd. 1, 1994, Bd. 2, 1999; Gundlach, Die öffentlich-rechtlichen Rundfunkunternehmen, 1998; Kruse, Ordnungspolitik im Rundfunk, in: Schenk/Donnerstag, Medienökonomie, 1989, 77, 89 f.; Brinkmann, Probleme der Marktregulierung des Rundfunks in der dualen Ordnung, in: Assmann u. a. (Hrsg.), Festgabe für Friedrich Kübler, 1997, $153 \mathrm{ff}$.

31 S. dazu Hoffmann-Riem (Fn. 28), 136 ff.

$32 \mathrm{Zu}$ solchen Hilfsdiensten und den damit verbundenen rechtlichen Problemen s. Gersdorf, Chancengleicher Zugang zum digitalen Fernsehen, 1998; Schulz/Held, Regulierung von Teleund Mediendiensten, 1997; Schulz/Seufert/Holznagel, Digitales Fernsehen, 1999; Thierfelder, Zugangsfragen digitaler Fernsehverbreitung, 1999; Leopoldt, Navigatoren - Zugangsregulierung bei elektronischen Programmführern im digitalen Fernsehen, 2002. 
Selbstverständlich sind nur Regulierungen gerechtfertigt, die zur Problemlösung geeignet, erforderlich und angemessen sind.

\section{Regulierte Selbstregulierung als Prototyp der Medienregulierung}

Medien-, insbesondere Rundfunkregulierung hebt sich zwar durch ihren besonderen Gegenstandsbereich von anderen staatlichen Regulierungen ab, ist aber hoheitliche Regulierung und kann daher auf die im Kern gleichen Regulierungskonzepte und -instrumente zugreifen, die auch in anderen Gegenstandsbereichen einsetzbar sind. Allerdings nötigt die besondere Sensibilität der Kommunikationsordnung gegenüber staatlichen Eingriffen - insbesondere der Grundsatz der Staatsfreiheit ${ }^{33}$ - zu besonderer Zurückhaltung. Gegenwärtig ist in vielen Bereichen eine Abnahme der Intensität staatlicher Regulierung und insbesondere eine Zurücknahme des Einsatzes hoheitlich-imperativer Instrumente zu verzeichnen. Solche Instrumente waren allerdings für den Medienbereich in der rechtsstaatlichen Demokratie ohnehin ohne besondere Relevanz. Für den Medienbereich ist vielmehr seit langem ein vorrangiges Vertrauen auf Selbstregulierung prägend, das allerdings durch eine regulative Umhegung dieser Selbstregulierung ergänzt wird. ${ }^{34}$ Selbstregulierung setzt das freiheitsbezogene Autonomieprinzip um; die regulatorische Umhegung solcher Selbstregulierung soll den Gemeinwohlbezug und das Prinzip der Rücksichtnahme auf andere sichern.

Medienregulierung ist ein Prototyp der hoheitlichen Regulierung gesellschaftlicher Selbstregulierung, geprägt insbesondere durch Rahmensetzung sowie Strukturvorgaben („Kontextsteuerung“) und Verhaltenspflichten, die auf die Beachtung eines rechtlich gesetzten Rahmens zielen (so auch z. B. Werberestriktionen, jugendschutzbezogene Bindungen sowie inhaltliche Programmvorgaben zur Vielfaltsicherung, nicht für konkrete Inhalte). ${ }^{35}$

Ein besonders wichtiger Modus der Selbstregulierung ist der ökonomische Markt. Der auf ihm maßgebende ökonomische Wettbewerb soll in einer privatwirtschaftlichen Medienordnung als Motor auch der kommunikativen Vielfalt wirken. Der Erfolgsmaßstab des ökonomischen Wettbewerbs ist in Geld ausgedrückt, zeigt sich also z. B. am Gewinn, an Marktanteilen, am Umsatz. ${ }^{36}$ Die Frage, ob ein solcher ökonomischer Wettbewerb ein hinreichender Garant auch eines publizistischen Wettbewerbs ist, bleibt umstritten. ${ }^{37}$ Das Bundesverfassungsgericht geht jedenfalls davon aus, dass im Bereich des Art. 5 Abs. 1 Satz 2 GG nicht der ökonomische, sondern der publizistische Wettbewerb

33 S. o. Fn. 12.

34 Dazu s. Holznagel, Regulierte Selbstregulierung im Medienrecht, Die Verwaltung Beiheft 4, 2001, $81 \mathrm{ff}$.

35 Derartige Regulierungskonzepte lassen sich weltweit beobachten, s. dazu Hoffmann-Riem, Regulating Media, 1996.

36 Vgl. die Definition bei Heinrich, Medienökonomie, Bd. 1, 1994, 95.

37 Bejahend Hoppmann, Meinungswettbewerb als Entdeckungsverfahren, in: Mestmäcker (Hrsg.), Offene Rundfunkordnung, 1998, 1163, 177 ff.; Wissenschaftlicher Beirat beim Bundesministerium für Wirtschaft und Technologie, Gutachten über eine „offene Medienordnung“, November 1999, Nr. 23 ff. Verneinend Stock, Rundfunkrecht und Wettbewerbsrecht im dualen Rundfunksystem, in: Hoffmann-Riem (Hrsg.), Rundfunk im Wettbewerbsrecht, 1988, 35 ff.; Rossen, Freie Meinungsbildung durch den Rundfunk, 1988, 35 ff.; Hoffmann-Riem, Rundfunkrecht neben Wirtschaftsrecht, 1991, 71 ff. 
maßgebend ist und dass letzterer aufgrund von Funktionsdefiziten des ökonomischen Marktes durch diesen allein nicht gesichert werden kann. ${ }^{38}$ Erfolgsmaßstab des verfassungsrechtlich bei Art. 5 GG maßgebenden Wettbewerbs sind publizistische Kategorien wie Meinungsvielfalt, kommunikative Entfaltung, plurale Ausgewogenheit u. ä. Ökonomischer Wettbewerb reicht nach dieser Konzeption als Grundlage der Funktionsfähigkeit der Medienordnung nur, wenn er auch zu einem funktionsfähigen publizistischen Wettbewerb führt, und zwar funktionsfähig auf dem durch die Verfassung vorgegebenen normativen Niveau. Rundfunkfreiheit ist der Auftrag und Rundfunkrecht ist der Versuch, die Maßgeblichkeit publizistischen Wettbewerbs auch gegen die Imperative ökonomischen Wettbewerbs - natürlich auch weiterhin gegen sonstige Begehrlichkeiten und Einwirkungsversuche staatlicher und nichtstaatlicher Machtträger - zu behaupten. ${ }^{39}$

Nicht zuletzt wegen des Grundsatzes der Staatsfreiheit kann und darf der Staat allerdings keine Verantwortung für bestimmte kommunikative Ergebnisse übernehmen (Erfüllungsverantwortung), sondern nur dafür, dass möglichst optimale Voraussetzungen der Kommunikationsversorgung aller (möglichst vieler) in einem Bereich bestehen, der vorrangig auf Eigenverantwortung der Akteure setzt. ${ }^{40}$ Anders formuliert: Kommunikationsinhalte (Programme) mit hinreichender Pluralität und auf einem den Möglichkeiten der Informations- und Wissensgesellschaft angepassten Qualitätsniveau lassen sich nicht staatlicherseits gebieten, sondern allenfalls ermöglichen. Medienrecht zielt auf solche „ermöglichenden“ Strukturen. Zur Sicherung von Kommunikationsfreiheit für alle (s. o. A I) gehört es, den Einsatz einseitiger ökonomischer und/oder publizistischer Macht zu verhindern, Manipulationsrisiken für die Rezipienten zu bannen und $\mathrm{Zu}$ gangshürden zu überwinden, die kommunikative Entfaltungsmöglichkeiten unangemessen behindern. In diesem Kontext lassen sich auch hoheitlich-imperative Instrumente unterstützend einsetzen, etwa Manipulationsverbote oder Öffnungsgebote (must-carry-rules, open access-Verpflichtungen u. ä.). ${ }^{41}$ Allerdings sind sie regelmäßig mit besonderen Implementationsschwierigkeiten verkoppelt.

Entlastend für die rundfunkrechtliche Regulierung kann die übrige Rechtsordnung wirken, die in ihrem privatrechtlichen Teil auch auf Selbstregulierung (Privatautonomie) baut. Neben dem Rückgriff auf Zivil-, Wirtschafts- und Urheberrecht ${ }^{42}$ kommt aber auch das hoheitliche Telekommunikationsrecht ${ }^{43}$ zum Zuge. Die allgemeine Rechtsordnung und das besondere Medienrecht können als wechselseitig nutzbare Teil-Auffang-

38 Vgl. BVerfGE 74, 297, 332; siehe auch 73, 118, 174.

39 Dass dieser Auftrag im Zuge der Kommerzialisierung der Medienordnung sich ändern und die Kommunikationsfreiheit grundlegend ihren Charakter wandeln kann, hat Tabbara eindrucksvoll an der Entwicklung der Kommunikationsfreiheit in den USA gezeigt, s. Tabbara, Kommunikations- und Medienfreiheit in den USA: Zwischen demokratischen Aspirationen und kommerzieller Mobilisierung, Diss. iur. Hamburg 2002.

$40 \mathrm{Zu}$ den unterschiedlichen Dimensionen staatlicher Verantwortungsübernahme siehe Schuppert, Die öffentliche Verwaltung im Kooperationsspektrum staatlicher und privater Aufgabenerfüllung: Zum Denken in Verantwortungsstufen, Die Verwaltung 31 (1998), 415 ff.

41 Ansätze dazu finden sich in dem $₫ 52,53 \mathrm{RStV}$. S. dazu etwa die Kommentierungen in Hartstein/Ring/Kreile/Dörr/Stettner, Rundfunkstaatsvertrag, Stand Mai 2001.

42 Dazu s. statt vieler Paschke, Medienrecht, 2. Aufl. 2001, 197 ff., $279 \mathrm{ff}$.

43 Aus der reichhaltigen Literatur s. statt vieler Trute/Spoerr/Bosch, TKG, 2001; Beck'scher-TKGKommentar, 2. Aufl. 2000. 
ordnungen verstanden werden. ${ }^{44}$ Allerdings ist bei dem Vertrauen auf dieses Zusammenspiel in Rechnung zu stellen, dass die rechtlichen Teilordnungen je eigenen Zielen folgen - so ist die allgemeine Rechtsordnung nicht speziell auf die Sicherung der Funktionsfähigkeit der Rundfunkordnung ausgerichtet - und entsprechend durch je eigene Rationalitäten geprägt sind und jeweils nur spezifische (also begrenzte) Leistungen erbringen können. Soweit aber die allgemeine Rechtsordnung auch zur Unterstützung der Funktionsfähigkeit der Rundfunkordnung beiträgt, ist sie ein wichtiges Hilfsmittel.

\section{IV.Steuerung unter Respektierung der Eigenrationalitäten der Adressaten}

Staatliche Regulierung zur Ausgestaltung der Medienordnung soll das Verhalten der Akteure so beeinflussen, dass die rechtserheblichen Zielwerte möglichst in deren autonomer Verantwortung erreicht werden. Rundfunkrecht will erwünschte Wirkungen unterstützen und unerwünschte möglichst vermeiden. Insofern ist es ein Mittel hoheitlicher „Steuerung“. ${ }^{45}$ Eine solche Steuerung im medienrechtlichen Sinne sind die Tätigkeiten des Staates, die auf die Erfüllung des Gewährleistungsauftrags aus Art. 5 Abs. 1 Satz 2 GG ausgerichtet sind, also der Konkretisierung und Ausgestaltung des Grundrechtes dienen. Untersuchungen über die Steuerungskraft von Recht - insbesondere aus dem Bereich der akteurszentrierten Handlungstheorie, der Institutionenökonomik und der Systemtheorie ${ }^{46}$ - verdeutlichen allerdings, dass die Fähigkeit des Rechts zur Erreichung erwünschter Ziele maßgebend durch die Handlungsrationalitäten (Eigenlogiken) in dem betreffenden Regelungsfeld bestimmt wird. Rechtliche Vorgaben werden am ehesten befolgt, wenn sie die Interessenvielfalt der Akteure durch Bereitstellung unterschiedlicher Optionen respektieren, sich dabei aber auf Optionen konzentrieren, die (auch) Gemeinwohlzwecke respektieren und die zugleich ein Verhalten der Akteure erlauben, das mit deren Eigeninteressen kompatibel ist oder das ihnen sogar kurz- oder langfristig Vorteile verspricht. Negativ formuliert: Eine rechtliche Steuerung gegen die Interessen der Betroffenen führt zu Ausweichreaktionen und Widerstand. Die Implementationschancen sind dann gering und können auch nur begrenzt durch repressive Mittel verbessert werden. Jedenfalls ist Repression in einer auf Selbstregulierung aufbauenden Ordnung ein Fremdkörper.

Es ist daher kein Zufall, dass die zur Implementation des Medienrechts im privatwirtschaftlichen Sektor eingesetzten Aufsichtsinstanzen - bei privatem Rundfunk etwa die Landesmedienanstalten ${ }^{47}$ - sehr zurückhaltend mit repressiven Sanktionen sind. Stattdessen sind sie um Kooperation bemüht, vertrauen auf informelle Problemlösun-

$44 \mathrm{Zu}$ diesem Konzept siehe die Beiträge in Hoffmann-Riem/Schmidt-Aßmann (Hrsg.), Öffentliches Recht und Privatrecht als wechselseitige Auffangordnungen, 1996.

45 Zur gegenwärtigen Steuerungsdiskussion siehe König/Dose, Klassifizierungsansätze staatlicher Handlungsformen, 1989; Schuppert, Verwaltungsrechtswissenschaft als Steuerungswissenschaft, in: Hoffmann-Riem/Schmidt-Aßmann/Schuppert (Hrsg.), Reform des Allgemeinen Verwaltungsrechts, 1993, 65 ff.; Benz, Kooperative Verwaltung, 1994; J.-P. Schneider, Kooperative Verwaltungsverfahren, Verwaltungsarchiv 1996, $38 \mathrm{ff}$. sowie - als systemtheoretische Perspektive - Willke, Supervision des Staates, 1997.

46 Zusammenfassende Darstellung dazu bei Hoffmann-Riem, Regulierung (Fn. 28), 158 ff.

$47 \mathrm{Zu}$ deren Aufsichtstätigkeit s. statt vieler Bumke, Die öffentliche Aufgabe der Landesmedienanstalten, 1995. 
gen und nutzen möglichst weitgehend „weiche“ Steuerungsinstrumente wie Beratung, Hinweise, gegebenenfalls die Inaussichtstellung sanktionierender Maßnahmen. ${ }^{48}$ Dies ist ein weltweit beobachtbarer Trend. ${ }^{49}$ Die Nutzung dieser weichen Steuerungshilfen ist insofern problematisch, als es zum einen keine Garantien des Erfolgs gibt, andererseits aber erhebliche Risiken der Zielverfehlung. So führt die kooperative Nähe zu den Regulierten zu dem Risiko der Übernahme ihrer einseitigen Perspektiven oder gar der Verstrickung in deren Handlungsimperative (capture). Andererseits bestehen gerade in der Kooperation Möglichkeiten der genaueren Erfassung der Interessen der Regulierten und damit zur Auswahl von Optionen, die sowohl die Befriedigung ihrer Interessen als auch die der Gemeinwohlinteressen erlauben.

\section{Herausforderungen an steuerndes Recht in der ausdifferenzierten Medienord- nung}

Es ist seit langem bekannt, dass die Medienregulierung nur begrenzt erfolgreich ist, und zwar auch für traditionellen Rundfunk. ${ }^{50} \mathrm{Da}$ aber auch ein begrenzter Erfolg besser ist als der Verzicht auf den Versuch einer Koppelung der Medienordnung mit Anforderungen an eine auch an Verfassungsprinzipien orientierte Funktionsfähigkeit folgt daraus nicht das Gebot eines Verzichts auf Medienregulierung. Allerdings muss versucht werden, sie jeweils unter Beachtung der aktuellen Rahmenbedingungen zu optimieren. Angesichts der Ausweitung und Ausdifferenzierung der Medienordnung stellen sich gegenwärtig viele neue Probleme, die veränderte Überlegungen zu ihrer rechtlichen Bewältigung nahe legen. Solche Problemfelder seien im Folgenden auswahlhaft benannt.

Aus der Sicht effektiven Grundrechtsschutzes sind heute vor allem Zugangssicherungen bedeutsam (s. schon o. II), so beispielsweise für die Kommunikatoren die der Zugänglichkeit von Produktionsstätten, von bestimmten Ereignissen - etwa massenattraktiven Sportveranstaltungen ${ }^{51}$ - oder des Zugangs zu wichtigen Programminhalten - etwa zu populären Kinofilmen. ${ }^{52}$ Wer die Zugänglichkeit steuert, kann die Wettbewerbsbedingungen und auch die publizistischen Wirkungschancen beeinflussen. Führt dies zu Machtasymmetrien, wird die regulatorische Verantwortung des grundrechtssichernden Gesetzgebers aktiviert.

Gleiches gilt für Einflussnahmen auf einen Zugang der Kommunikationsangebote zu Rezipienten. Technisch vermittelte und marktwirtschaftlich angebotene Kommunikation kennt unweigerlich Zugangshürden. Der objektiv-rechtliche Grundrechtsauftrag zielt darauf, diese so zu gestalten, dass die Freiheitlichkeit der Kommunikation für die Rezipienten nicht leidet. Besondere Aufmerksamkeit verdienen insofern Manipulationsmöglichkeiten.

In der modernen Zeit der Informationsgesellschaft ermöglichen es die Medientechnologien und die vielschichtigen Medieninfrastrukturen, auf subtile, zum Teil aber auch

48 Siehe dazu Arbeitsgemeinschaft der Landesmedienanstalten in der Bundesrepublik Deutschland, Medienregulierung im Wandel - zum Rang und zur Rolle der Landesmedienanstalten. Ein Positionspapier der ALM vom 23. März 1999; Baars, Kooperation und Kommunikation durch Landesmedienanstalten. Eine Analyse ihres Aufgaben- und Funktionsbereichs, 1999.

49 Siehe dazu die Beobachtungen in Hoffmann-Riem (Fn. 35), 1996.

50 Nähere Belege - mit weltweitem Anschauungsmaterial - bei Hoffmann-Riem (Fn. 35).

51 Dazu siehe $B \operatorname{VerfGE} 97,228 \mathrm{ff}$.

52 S. dazu Ladeur, Rundfunk und Fernsehen 1998, 5 ff. 
auf nichtsubtile Art zu manipulieren. Es ist ein Irrglaube zu meinen, dass allein der Wettbewerb der Medien untereinander die Rezipienten vor Manipulation schützt. Es gibt zu viele Anreize manipulativen Vorgehens. So sehen sich die Medienunternehmen in einem Wettbewerb um eine knappe Ressource: die Aufmerksamkeit der Rezipienten/Konsumenten ${ }^{53}$ und sind bemüht, diese Aufmerksamkeit zu erhalten, und gegebenenfalls versucht, dafür auch manipulativ-suggestive Techniken einzusetzen.

Besondere Möglichkeiten dazu bieten die so genannten Navigatoren. Angesichts der mit der Digitalisierung und der Datenreduktion möglichen Vervielfachung von Übertragungskapazität und entsprechender Angebote erhält die Orientierung des Rezipienten über das Programmangebot und die „Ansteuerung“ der Angebote eine bedeutende Aufgabe, die durch dafür konzipierte Dienste, insbesondere Navigatoren, bewältigt werden kann. ${ }^{54}$

Navigatoren übernehmen wie eine „elektronische Programmzeitschrift“, aber viel stärker mit der realen Nutzung verknüpft, die Benutzerführung und ermöglichen die Orientierung sowie den Zugriff zu Programmen und anderen Anwendungen. Damit besteht ein Risiko der Diskriminierung bestimmter Dienste, etwa durch ungünstige Platzierung, Gestaltung, Verzeichnisbildung oder die Art der Verknüpfungsmöglichkeiten. Wenn Navigationsdienste Nutzer auf bestimmte Kommunikationswege verweisen, Kommunikationsangebote in bestimmter Reihenfolge aufrufen u. ä., dann sind damit auch Möglichkeiten verknüpft, den Nutzer gezielt zu lenken und bestimmten Kommunikationsangeboten Vorteile vor anderen zu verschaffen. So ist es beispielsweise für die Chancen, als Fernsehsender erfolgreich zu sein, wichtig, wie der Nutzer auf dessen Programm zugreifen kann. Es macht für die Erfolgschancen einen Unterschied, ob beim Einschalten des Navigationssystems zunächst auf dieses Programm - oder nur auf andere Programme anderer kommerzieller oder nur die öffentlicher Veranstalter - verwiesen wird. Auch macht es einen Unterschied, ob Fernsehprogramme direkt angewählt werden können oder ob dies nur über mehrere Schritte möglich ist.

Werden Navigationssysteme und Suchportale zudem dazu genutzt, die im Abrufvorgang ablesbaren Interessen der individuellen Nutzer aufzunehmen und zu speichern, Datenmuster zu analysieren und Nutzerprofile zu erstellen („collaborative filtering“), dann kann dies z. B. als Grundlage eingesetzt werden, die zukünftig vermittelten Angebote speziell auf die individuellen Nutzerinteressen auszurichten - Fußballfreunden also zunächst Fußballprogramme anzubieten, Krimifreunden Krimis und zugleich Werbebotschaften und Verkaufsaufforderungen (E-Commerce) auf die Interessen der je individuellen Konsumenten auszurichten. Diese werden häufig sogar damit einverstanden sein. Dennoch ist unübersehbar, dass hier erhebliche Beeinflussungsmöglichkeiten bestehen. Die modernen Technologien schaffen nicht nur Macht zur Lenkung des Kommunikationsflusses, sondern auch zur Datenerhebung, -verarbeitung und -weiternutzung. Regelmäßig wissen Nutzer nicht, welche Software in einem Navigationssystem verwendet wird oder auf dem Computer installiert ist und wieweit sie dazu genutzt wird, Schritte der Kommunikationsnutzung zu speichern, Datenmuster zu erfassen, die

53 Vgl. dazu Franck, Ökonomie der Aufmerksamkeit, 1998; Rötzer, Aufmerksamkeit als Medium der Öffentlichkeit, in: Maresch/Werber (Hrsg.), Kommunikation, Medien, Macht, 1999, 35 ff.; Schmidt, Kalte Faszination: Medien, Kultur, Wissenschaft in der Mediengesellschaft, 2000, $234 \mathrm{ff}$.

54 S. dazu Gersdorf (Fn. 32), 69 ff.; Schulz/Senfert/Holznagel (Fn. 32), 81, 102 ff.; Thierfelder (Fn. 32), 144 f., 147 ff., 160 f. 
entsprechenden Informationen an Dritte weiterzugeben und für zukünftige Steuerungsakte zu nutzen.

Dies illustriert, dass neue Technologien auch neue Macht - hier Lenkungs- und Filtermacht - vermitteln können. Liegen die entsprechenden Navigationssysteme in den Händen von Wirtschaftsunternehmen, die zugleich Inhalte von Medien, also Programme, bereitstellen, dann gibt die Möglichkeit der Kombination dieser Aktivitäten zusätzliche Marktchancen. Die Versuchung liegt nahe, die Navigationssysteme so auszugestalten, dass die von dem Unternehmen selbst stammenden Programme bevorzugt rezipiert werden. Kommerzielle Interessen von Werbeunternehmen können ferner die Versuchung bedingen, die Angebots- und Auswahlmacht zu nutzen, um solchen Programmen bevorzugte Rezeptionschancen zukommen zu lassen, die auch der Werbewirtschaft zusagen, etwa weil sie ein für den Absatz der beworbenen Produkte günstiges Programmumfeld bereitstellen. Auf der Verlustseite kann die Medienvielfalt zu verbuchen sein.

Eine weitere, insbesondere bei Pay-TV-Programmen beobachtbare Erscheinung ist die Bündelung von Programmen zu Programmpaketen, die den Rezipienten angeboten werden (packaging) und die sie häufig auch nicht aufgeteilt (entbündelt) beziehen können. Durch Programmpaketbildung wird eine besondere Vermarktungsform geschaffen. Neben Pay-TV-Programmen können auch Free-TV-Programme, Medien-, Teleoder Sprachtelefondienste in ein Bouquet aufgenommen werden. Dadurch können z. B. marktbeherrschende Anbieter aus dem Teledienste- bzw. Sprachtelefondienstemarkt Einfluss auf den Rundfunksektor gewinnen. Insofern ist zumindest eine Vorkehrung dafür wichtig, dass ein Anbieter, der bei der Bündelung und Vermarktung von Programmen eine marktbeherrschende Stellung innehat, Anbieter, die einen solchen Dienst nachfragen, weder unmittelbar noch mittelbar unbillig behindert oder gegenüber gleichartigen Anbietern ohne sachlich gerechtfertigten Grund unmittelbar und mittelbar unterschiedlich behandelt.

Solche Beispiele illustrieren, dass Strukturen und Verhaltensweisen auch in den der Medienveranstaltung vor-, neben- und nachgelagerten Bereichen mit dem Blick auf Art. 5 Abs. 1 Satz 2 GG bedeutsam sind. Dass der Geltungsbereich dieser Grundrechtsnorm sich auf diese Bereiche erstreckt, hat das Bundesverfassungsgericht bisher zwar noch nicht umfassend, wohl aber für verschiedene Beispielsfelder bejaht (Stichworte: Pressegrosso, Kurzberichterstattung u. a.). ${ }^{55}$ Der Gesetzgeber beginnt, das Problem zu erkennen, also seinen objektiv-rechtlichen Grundrechtsauftrag zur Ausgestaltung der Freiheitlichkeit der Kommunikation wahrzunehmen. Vorkehrungen zur Sicherung eines chancengleichen, angemessenen und nicht diskriminierenden Zugangs zu einigen Diensten enthält z. B. $\int 53 \mathrm{RfStV}$, wenn auch noch in unzureichender Weise. ${ }^{56}$

In den im Aufbau befindlichen Multimedia-Netzwerken haben sich neue Strukturen herausgebildet, die mit den geschilderten Vermachtungs- und Missbrauchsrisiken verbunden sind. Dem Gewährleistungsgesetzgeber des Art. 5 Abs. 1 Satz 2 GG dürfen die Entwicklungen in diesen Sektoren nicht gleichgültig sein. Die Regulierung aber ist schwierig, wenn sie nicht mit dem Risiko verbunden sein soll, neue Entwicklungen zu unterbinden oder zwar in Deutschland Restriktionen vorzusehen, diese aber aufgrund

$55 \mathrm{Vgl}$. BVerfGE 77, 346, 354; 83, 238, 312 ff.; 97, 228, 267.

$56 \mathrm{Zu}$ den mit $\int 53$ RfStV verbundenen Problemen s. Schulz/Küblers, Konzepte der Zugangsregulierung für das digitale Fernsehen, 2000; Schulz, K \& R 2000, 9 ff.; Thierfelder, Zugangsfragen digitaler Fernsehverbreitung, 1999; Leopoldt (Fn. 32). 
der globalen Aktivitäten auswärtiger Veranstalter und Diensteanbieter nicht oder nur begrenzt durchsetzen zu können.

Wichtig dürfte insbesondere die Sorge dafür sein, dass die verfügbaren Angebote nicht nur nach kommerziellen Imperativen gestaltet sind. Insofern erhält die ältere Frage neue Bedeutung, ob die mit dem dualen Rundfunksystem verbundene Idee struktureller Diversifikation $^{57}$ auch bei anderen als traditionellen Rundfunkdiensten nutzbar gemacht werden kann. Dies würde beispielsweise dazu führen können, die Bereitstellung solcher Dienste auch gemeinwirtschaftlichen Veranstaltern, darunter auch den öffentlich-rechtlichen Rundfunkanstalten, zu gestatten. ${ }^{58}$ Angesichts der Dynamik der Märkte, der Unvorhersehbarkeit neuer technologischer Entwicklungen und der Variabilität von Nutzerinteressen und -gewohnheiten muss die Medienordnung jedenfalls auf ein hohes Maß an Lernfähigkeit, zugleich auch Revisionsoffenheit, ausgerichtet sein. Das Konstruktionsprinzip der dualen Rundfunkordnung ist zwar nicht als einzig mögliches verfassungsrechtlich vorgegeben, entspricht aber in besonderer Weise einer Konzeption, die weitestgehend Privatwirtschaftlichkeit zulässt und nutzt, aber die Pflicht zur Vorsorge dafür einlösen muss und über den öffentlich-rechtlichen Rundfunk einzulösen versucht, dass die mit Privatwirtschaftlichkeit als alleiniger Wirtschaftsform verbundenen Defizite ausgeglichen werden. Ändern sich die Realbedingungen und damit auch die Gefährdungen, gibt es Anpassungsbedarf bei den zur Verwirklichung der unverändert fortbestehenden Ziele der Kommunikationsordnung vorgesehenen Instrumenten. Der objektiv-rechtliche Auftrag zur Sicherung der Funktionsfähigkeit der Medienordnung - also zum Erhalt der Freiheitschance für alle - wandert mit, wenn sich die Gefahrenursachen, also auch die Regelungsprobleme, in neue Bereiche der Kommunikation und der entsprechenden Netzwerke verlagern.

\section{Umgang mit Risiken der „Zensur“ beim Informationszugang}

Der Kampf um die Freiheit hatte - wie eingangs schon dargestellt - immer etwas mit dem Kampf gegen Macht zu tun. Freiheitsrechte zielen auf die Bereitstellung von Mitteln gegen Machteinsatz, vor allem gegen Machtmissbrauch. Wenn das Risiko des Machtmissbrauchs von privaten Unternehmen ausgeht, dann setzt eine freiheitliche Ordnung Schutz auch vor ihm voraus. Deshalb ist wichtig, dass Grundrechte nicht nur Abwehrrechte gegen den Staat, sondern auch objektiv-rechtliche Schutzaufträge an ihn enthalten. Dies gilt auch für das Zensurverbot (Art. 5 Abs. 1 Satz 3 GG), gewissermaßen den Prototyp eines klassischen Abwehrrechts.

Historisch gesehen ist das Zensurverbot als Mittel gegen staatliche und kirchliche Zensur entstanden. ${ }^{59}$ Die Zensurfreiheit war im 19. Jahrhundert der wesentliche Kern der Pressefreiheit. Als Gefährder der Freiheit erschien in erster Linie der Staat. Das Zensurverbot schafft daher ein Abwehrrecht des Einzelnen gegen den Staat. Heute aber gibt es weitere Gefahrenträger. Dies sei abschließend am Beispiel des Internet illustriert.

Das Internet ermöglicht den Zugang zu vielfältigen Informationen aller Art und un-

57 Dazu s. Hoffmann-Riem, Erosionen des Rundfunkrechts, 1990, 38 f.; ders., Staatswissenschaften und Staatspraxis 1991, 412 f.; ders., Regulierung (Fn. 28), 67 ff., 292 ff.

$58 \mathrm{Ob}$ dies schon gegenwärtig zulässig ist, ist in der Literatur umstritten, siehe dazu HoffmannRiem, Regulierung (Fn. 28), 234 ff. m.w.Hinw.

59 Zur Geschichte des Zensurverbots s. Fiedler, Die formale Seite der Äußerungsfreiheiten, 1999; Robde, Die Nachzensur in Art. 5 Abs. 1 Satz 3 GG, 1997. 
terschiedlicher Herkunft. Eine Begleiterscheinung ist, dass es Inhalte gibt, die gesellschaftlich als unerwünscht gelten, wie z. B. Pornografie, rechtsextremistische Propaganda oder Anleitungen zum Bombenbau. Es darf daher nicht überraschen, dass eine intensive Diskussion darüber entstanden ist, wie solche unerwünschten Inhalte aus dem Internet herausgehalten werden können ${ }^{60}$. Es gibt in Europa einen weiten gesellschaftlichen Konsens, dass z. B. Jugendschutz legitim ist, dass Terrorismus bekämpft werden muss und dass es ein legitimes Anliegen demokratischer Staatsordnungen ist, politisch extremistische Inhalte abzuwehren.

Das Internet wird nicht vom Staat verwaltet. In der Selbstverwaltung des Internet haben große Unternehmen starken Einfluss, beispielsweise die so genannten Provider wie America Online (AOL). Das Internet ist so organisiert, dass es aufgrund seiner spezifischen Netzstruktur nur begrenzt einer Kontrolle unterzogen werden kann. ${ }^{61}$ Jedenfalls ist der Nationalstaat als Kontrolleur weitgehend ohnmächtig. Selbst wenn der Staat versuchen wollte, die Inhalte zu kontrollieren, so wäre er nur begrenzt als Träger von Zensur erfolgreich. In diese Lücke sind zum Teil private Unternehmen, insbesondere die Provider, gerückt, die angefangen haben, das Internet auf unerwünschte Inhalte zu durchkämmen oder durchkämmen zu lassen und z. B. aufgefundene Pornografie oder politisch extremistische Inhalte zu sperren. Nach deutschem Recht verlangt der Gesetzgeber die Sperrung von Inhalten durch Provider, soweit sie von rechtswidrigen bzw. strafbaren Inhalten Kenntnis haben (vgl. $\iint 5$ TDG, MDStV).

Blickt man auf den traditionellen Gehalt des Zensurverbots, dann haben systematische Inhaltskontrollen und Filterungen der Verbreitung von Inhalten durch private Provider nichts mit Zensur i. S. des Art. 5 Abs. 1 Satz 3 GG zu tun. Es geht ja nicht um Zensurmaßnahmen des Staates. Wird der übergreifende Sinn des Verbots der Zensur aber in der Verhinderung einer Lähmung des Geisteslebens und des manipulativ-steuernden Zugriffs auf Kommunikationsinhalte gesehen ${ }^{62}$, dann liegt die Wertung nahe, auch in der Tätigkeit von privaten Filterinstanzen eine ähnliche Gefahr für die Freiheitlichkeit der Kommunikation zu sehen, wie sie früher vom Staat ausging. Heute ist der Staat demokratisch organisiert und rechtsstaatlich vielfältig kontrolliert. Über entsprechende verantwortungssichernde Strukturen und demokratische Kontrollvorkehrungen verfügen Privatunternehmen, auch die Provider im Internet, nicht. Es ist daher nicht ausgeschlossen, dass die private Steuerungs- und Filtermacht aufgrund der geringen Kontrollmöglichkeiten gefährlicher für die Freiheitlichkeit der Kommunikation ist als eine entsprechende Kontrolle durch den Staat.

Werden Kontrollmaßnahmen in systematischer Weise durchgeführt - dazu gibt es schon vielfältige Möglichkeiten ${ }^{63}$-, dann besteht das Risiko, dass bestimmte Inhalte ganz aus dem Internet verschwinden, jedenfalls soweit nicht auf andere Provider ausgewichen werden kann. Nun wird vermutlich kaum jemand etwas dagegen einwenden, wenn strafbare Inhalte verhindert werden. Wer aber garantiert, dass die Filterung von Kommunikationsinhalten sich darauf beschränkt - ganz abgesehen davon, dass es häu-

60 S. statt vieler Hornig, Möglichkeiten des Ordnungsrechts bei der Bekämpfung rechtsextremistischer Inhalte im Internet, ZUM 2001, 846 ff. m. w. Hinw.

61 Zur Funktionsweise des Internet s. statt vieler Beck/Prinz, Ökonomie des Internet, 1999. Zu den Rechtsfragen der Haftung s. statt vieler Freytag, Haftung im Netz, 1999.

62 I.d.S. Hoffmann-Riem in AK-GG, 3. Aufl. 2001, Rn. 89 ff. zu Art. 5 Abs. 1, 2 GG.

63 Als knapper Überblick über unterschiedliche technische Sperr- und Filtersysteme s. Vielhaber, Neuer Schutz vor neuen Gefahren? Jugendschutz im Internet, MMR Beilage 2001/9, 16, $18 \mathrm{f}$. 
fig auch schwierig ist, die Grenze des Strafbaren zu bestimmen? Die - gegenwärtig immer weiter ausgebaute - Klassifizierung von Angebotsinhalten als Grundlage der Filterung oder als Entscheidungshilfe für die Nutzer setzt Wertungen voraus. Diese können in weltanschauliche Fragebereiche hineinwirken oder sonst wie folgenreiche Vorentscheidungen fordern. Wer garantiert, dass nicht auch Inhalte ausgefiltert werden, die den Providern politisch unerwünscht sind oder die z. B. Kritik an wirtschaftlichen Vorgängen oder an der wirtschaftlichen Macht des Providers üben? Jedenfalls scheint eine Garantie vor Machtmissbrauch nicht gegeben zu sein, wenn nicht auch hier die ordnende Kraft des Rechts wirksam wird. Insoweit ist es eine wichtige Frage der Gegenwart und Zukunft, ob das als Verbot staatlicher Zensur entstandene Zensurverbot unter den veränderten Rahmenbedingungen umgedacht und zur Sicherung werden muss, Schutz auch vor zensurähnlichen Akten durch private Wirtschaftsunternehmen zu gewähren. Schutz mit Hilfe staatlichen Rechts aber setzt voraus, das Zensurverbot des Art. 5 Abs. 1 Satz 3 GG auch als objektiv-rechtlichen Grundrechtsauftrag an den Staat zu verstehen, Vorkehrungen gegen den Aufbau privater Zensurmacht zu schaffen, etwa Transparenz- und Kontrollvorkehrungen bei zensurähnlichem Verhalten von Providern vorzusehen.

\section{Fazit}

Die Zukunft der Freiheitsrechte wird auch davon abhängen, dass bei neuen Erscheinungen immer wieder gefragt wird, ob die schon erfolgte rechtliche Gestaltung des Freiheitsbereiches ausreicht, um unter heutigen Bedingungen Ziele zu verwirklichen, die auch schon gestern wichtig waren. Der rasante Umbruch auf dem Weg zur Informationsgesellschaft legt Antworten auf diese Frage dringend nahe. Die Ziele des Freiheitsschutzes sind in der Neuzeit weitgehend gleich geblieben, die Wege zur Zielerreichung aber müssen immer wieder auf die je aktuellen Verwirklichungsbedingungen abgestimmt werden. Daher bleibt der objektiv-rechtliche Gehalt der kommunikationsbezogenen Grundrechte auch in der Gegenwart und Zukunft wichtig, und zwar zur Gewährleistung subjektiven Freiheitsschutzes für möglichst alle. Den Schutz des Rechts benötigen vor allem Machtschwache. 